\title{
COALITIONS OF THE WILLING: COYUNTURA, CONTEXTO Y PROPIEDADES. UN PRIMER ESBOZO
}

\begin{abstract}
Alejandro RoDILES*
RESUMEN: La última guerra en Iraq dio a conocer el fenómeno llamado coalition of the willing. Sin embargo, la alianza militar ad hoc liderada por Estados Unidos de América no fue su primera manifestación, ni se trata del único tipo de coalición que actúa en la escena internacional. Si bien podemos ubicar los primeros antecedentes a principios del siglo XX, las coalitions continúan evolucionando, según lo demandan los nuevos temas globales que afrontan. Este artículo es un intento por identificar sus propiedades esenciales. Al mismo tiempo, se parte de la necesidad de ponerlas en contexto, con el fin de mostrar que, a pesar de su naturaleza política, inciden decididamente en el sistema de fuentes del derecho internacional y en las legislaciones nacionales.

ABSTRACT: The last war in Iraq has brought to light the phenomenon called coalition of the willing. Nevertheless, the ad hoc military alliance led by the United States has not been its first manifestation nor is it the only type of coalitions acting on the international scene. Although their first appearances can be found at the beginning of the XXth century, coalitions continue to evolve as demanded by the new global issues they face. This article is an attempt to identify their essential properties. At the same time, it endeavours to put them into context in order to demonstrate that, despite their political nature, coalitions have a concrete impact on the sources of international law, as well as on national legislations.

RÉSUMÉ: La dernière guerre en Irak a mis en lumière le phénomène appelé coalition of the willing. Toutefois, cette alliance militaire ad hoc sous le leadership des Etats-Unis n'a pas été sa première manifestation; d'ailleurs, il ne s'agit pas du seul type de coalition qui existe sur la scène internationale. Si l'on retrouve les premières manifestations du phénomène au début du XXème siècle, les coalitions ont évolué en fonction des nouveaux sujets de l'agenda globale qu'elles cherchent à affronter. Cet article essaie d'identifier ses caractéristiques essentielles. Aussi, il a été considéré nécessaire de mettre les différentes coalitions dans leur propre contexte. Ceci démontrera que, malgré leur nature politique, les coalitions ont des effets réels sur le système des sources du droit international ainsi que sur les législations nationales.
\end{abstract}

* Profesor de derecho internacional público y derecho europeo en la Facultad de Derecho de la UNAM. 
Ningún concepto de dominio legítimo puede ser definido en alguna forma que no sea referido a la facultad de mandar.

Max WeBER

\begin{abstract}
Sumario: I. Coyuntura. II. Contexto. III. Propiedades. IV. Conclusiones.
\end{abstract}

\title{
I. COYUNTURA
}

El uso no autorizado de la fuerza por parte de los Estados Unidos de América (EUA) y sus aliados contra Iraq cuestionó seriamente la eficiencia de las Naciones Unidas y, con ello, la eficacia de las normas fundamentales de la carta. Pero, al mismo tiempo, la tercera guerra del Golfo Pérsico provocó, aún antes de desatarse, "la mayor movilización mundial en la historia". ${ }^{1}$ Y si bien esta manifestación global se dirigió directamente contra la violencia militar, detrás de ello se denotaba un reclamo más general en defensa del derecho internacional. ${ }^{2}$ El desafío de la superpotencia hacia los procesos multilaterales establecidos, en virtud de su interpretación sobre la legítima defensa preventiva ${ }^{3}$ y la creación de alian-

1 El 15 de febrero de 2003 se manifestaron varios millones de personas en las principales ciudades del mundo en contra de la guerra en Iraq. Una lista detallada de las concentraciones que tuvieron lugar ese día puede encontrarse en http://es.wikipedia.org/wiki. Manifestaciones_mundiales_contra_la_guerra_de_Iraq.

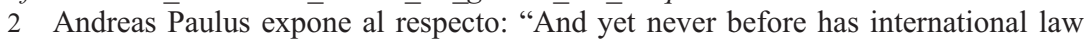
been so popular. Millions of peaceful protesters were marching to uphold it". Cfr. Paulus, Andreas, "The War Against Iraq and the Future of International Law: Hegemony or Pluralism?”, Michigan Journal of International Law, núm. 25, 2004, pp. 691-733.

3 Es preciso distinguir entre legítima defensa anticipada (anticipatory self-defense) y legítima defensa preventiva (preemptive self-defense). La primera se refiere a la respuesta militar proporcional frente a una amenaza inminente, que no encuentre otro medio de solución, en tanto la segunda es la acción militar frente a una amenaza latente, que podría ocurrir ( $c f r$. Informe del Secretario General de las Naciones Unidas, Un concepto más amplio de libertad: desarrollo, seguridad y derechos humanos para todos, A/59/2005, 21 de marzo de 2005, párrs. 122-126; así como el Informe del Grupo de Alto Nivel sobre las amenazas, los desafíos y el cambio, Un mundo más seguro: la responsabilidad que compartimos, A/59/565, 2 de diciembre de 2004, párrs. 188-192). A pesar de lo sostenido en ambos informes, existe controversia doctrinaria respecto a la admisibilidad de la legítima defensa anticipada y varios Estados se oponen aún a todo tipo de interpretación amplia del artículo 51 de la carta. Por su parte, la tesis de la 
zas al margen de los foros institucionalizados, se topó no sólo con el rechazo de la mayoría de los Estados sino, quizá por primera vez, de la opinión pública mundial. A la postre podemos decir que, a pesar de todo, la guerra contra Iraq tuvo el efecto sumamente positivo de difundir un debate hasta entonces muy exclusivo acerca del presente y futuro papel del derecho internacional. ${ }^{4}$ En gran parte, el debate consiste en las respuestas que tendrá que desarrollar la comunidad internacional organizada ante los retos de un mundo cada vez más interrelacionado. De ahí que la reforma de las Naciones Unidas sea un aspecto clave al respecto. Por otro lado, aunque estrechamente vinculado con lo anterior, se encuentra el desafío que representa el poder hegemónico estadounidense para los fundamentos y el desarrollo del derecho de gentes. ${ }^{5}$

La discusión en torno a la legalidad y legitimidad de la guerra en Iraq es un fiel reflejo de ambos aspectos de este debate fundamental, toda vez que el aparente problema que sirvió de pretexto para abandonar las vías institucionales, $i$. e. la incapacidad de respuesta del Consejo de Seguridad (CSNU) ante las amenazas a la paz y seguridad internacionales, ${ }^{6}$

legítima defensa preventiva, también conocida como 'doctrina Bush', es ampliamente rechazada por la doctrina y por la gran mayoría de los Estados. Asimismo, cabe reiterar que esta tesis no fue presentada por EUA ante el Consejo de Seguridad (CSNU) como argumento jurídico a favor de la intervención en Iraq, sino que representa una visión a futuro de la administración Bush sobre la evolución que tendrá que experimentar el principio de la proscripción del uso de la fuerza, con el fin de adaptarse a los nuevos retos globales, como la proliferación de armas de destrucción masiva (ADM) y el terrorismo. En este contexto, el fenómeno que nos ocupa podría desempeñar un papel muy importante, pues es de esperarse que las coalitions se conviertan en el instrumento idóneo para usar la fuerza de manera preventiva, ya que, aparte de las obvias ventajas estratégicas, sirven para legitimar estas acciones.

4 Así, por ejemplo, Georg Nolte: “A más tardar a partir de la guerra de Iraq de 2003, se desató una discusión fundamental sobre el rol del derecho internacional en general y la necesidad de reformar a las Naciones Unidas en particular, que por mucho traspasó las fronteras de los círculos especializados". Véase Nolte, Georg, "Sobre crisis y crecimiento del derecho internacional en sesenta años de Naciones Unidas", Anuario Mexicano de Derecho Internacional, supra p. 228.

5 Un panorama amplio sobre este tema puede encontrarse en Byers, Michael y Nolte, Georg (eds.), United States Hegemony and the Foundations of International Law, Cambridge, Cambridge University Press, 2003.

6 En términos generales, el problema de la falta de capacidad de respuesta eficaz ante los principales riesgos globales por parte de las Naciones Unidas, y del CSNU en particular, no es aparente; pero sí lo fue en el caso concreto de la supuesta posesión iraquí de ADM. 
fue prontamente rebasado por otro conflicto, que si bien no es nuevo, adquirió nuevas dimensiones después del 11 de septiembre de 2001: la inobservancia de las reglas básicas del juego por parte del jugador más poderoso.

El discurso radicalizado de la actual administración estadounidense, ya clásicamente sintetizado en aquella frase que pronunciara el presidente George W. Bush ante el congreso unos días tras los atentados terroristas: "Either you are with us, or you are with the terrorists", ${ }^{7}$ reflejó un encrudecimiento de las tendencias unilateralistas estadounidenses, despertando así el interés público mundial sobre los alcances del desafío que representa un hegemón lastimado para el multilateralismo y el derecho internacional.

Es cierto que el acontecimiento que publicitó el término 'coalition of the willing', ${ }^{8} i$. e. la alianza militar ad hoc de un grupo de países liderados por EUA para desarmar y derrocar el régimen de Saddam Hussein, no representa un fenómeno típico del unilateralismo tradicional. Más bien forma parte de un multilateralismo selectivo, o como dijo el antiguo jefe de Planeación Política del Departamento de Estado, Richard Haass, de un multilateralism à la carte, ${ }^{9}$ basado en un enfoque de escoger, caso por caso, a los miembros del club, según el famoso 'pick and choose ap-

7 The White House, Address to a Joint Session of Congress and the American People, Office of the Press Secretary, Press Release, 20 de septiembre de 2001, en http://www.whitehouse.gov/news/releases/2001/09/20010920-8.html.

8 A la fecha no existe un término comúnmente aceptado en español, lo cual refleja su reciente recepción en el discurso público y en el mundo académico hispanohablante. Suele ser traducido como 'coaliciones voluntarias', 'de buena voluntad', 'de voluntarios' o 'de los dispuestos'. Los primeros tres términos deben ser descartados definitivamente, pues no transmiten la intensión del concepto en inglés. 'Coaliciones de los dispuestos' es, sin duda, una traducción correcta, aunque no convence totalmente, en tanto que 'alianzas ad hoc' sólo transmite parcialmente la idea. Por lo anterior, seguiré usando el término en inglés, su forma abreviada ('coalitions') o, simplemente, 'coaliciones'.

9 Una de las primeras versiones sobre este concepto puede observarse en Haass, Richard N., The Reluctant Sheriff: The United States after the Cold War, Council on Foreign Relations, 1997. Sin referirse concretamente al término, la idea es recurrente a lo largo de los discursos que pronunció como asesor principal de Colin Powell; véase, por ejemplo: Haass, Richard N., Planning Policy in Today's World, Remarks at the Kennan Institute 2003, Annual Dinner, 22 de mayo de 2003, en http://www.state.gov/s/p/rem/ 2003/20910.htm; Haass, Richard N., From Reluctant to Resolute: American Foreign Policy after September 11, Remarks to the Chicago Council on Foreign Relations, 26 de junio de 2002, en http://www.state.gov/s/p/rem/11445.htm. 
proach'. Al respecto, el propio Haass menciona lo siguiente: "Multilateralism comes in many forms. Multilateral cooperation is most successful when it is built on real convergence of interests and values... when the United Nations or other bodies are unwilling or unable to move against dire threats, we reserve the right to act in less encompassing alliances or flexible, ad hoc coalitions of the willing". ${ }^{10}$

Como se desprende del discurso oficial de la administración Bush, EUA se concibe a sí mismo como el núcleo natural que atrae a los países "capaces y dispuestos"11 de defender las mismas causas; no se trata de alianzas entre iguales, sino de agrupaciones reunidas en torno al líder (o sheriff, según Haass): "Call it the 'posse' model, in wich we assemble 'coalitions of the willing' to deal with the issue at hand". ${ }^{12}$ En dicho sentido, cabría cuestionarse si no estamos en realidad ante la presencia de un "unilateralismo disfrazado". ${ }^{13}$ Por otro lado, la afirmación de que esta concepción es sólo una adaptación del multilateralismo a los nuevos riesgos globales, con el fin de que pueda hacerles frente de manera más eficiente, ${ }^{14}$ no es, a priori, desdeñable. Sea como sea, es incuestionable que

10 Haass, Richard N., Planning Policy..., cit., nota anterior. El énfasis es mío.

11 Así en la Estrategia de Seguridad Nacional 2002: “America will implement its strategies by organizing coalitions — as broad as practicable — of states able and willing to promote a balance of power that favours freedom" (The National Security Strategy of the United States of America, septiembre de 2002, p. 25, en http://www.whitehouse. gov/nsc/nss/2002/index.html).

12 Haass, Richard N., From Reluctant to Resolute..., cit., nota 9. Énfasis añadido.

13 Risse, Thomas, Effective Multilateralism vs. Coalitions of the Willing-The Crisis of the Transatlantic Security Community, Hellenic Center for European Studies, 3 a 5 de junio de 2005, en http://www.ekem.gr/archives/2005/06/effective_multi.html.

14 De ahí que el gobierno estadounidense — a veces secundado por el británicohable también de 'multilateralismo efectivo', especialmente en el contexto del combate contra la proliferación de ADM ( $c f r$. The White House, US-UK Joint Statement on Multilateralism, Effective Multilateralism to Build a Better World, Press Release, Office of the Press Secretary, 20 de noviembre de 2003, en http://www.whitehouse.gov/news/releases/2003/11/20031120.html; así como Rademaker, Stephen G., Acting Assistant Secretary, International Security and Nonproliferation, Remarks at the Conference on Disarmament, Rising to the Challenge of Effective Multilateralism, Ginebra, Suiza, mayo de 2006, en http://www.state.gov/t/isn/rls/rm/66419.htm). Sin embargo, en el marco de la Unión Europea, y particularmente impulsado por el alto representante de la Política Exterior y de Seguridad Común, Javier Solana, el concepto 'multilateralismo efectivo' ha recibido una connotación distinta, pues si bien se acepta el riesgo de la parálisis internacional, el énfasis está puesto en el fortalecimiento de las organizaciones internacionales existentes, en pleno apego al derecho vigente ( $c f r$. The Council of the Euro- 
representa un desafío para las formas y procesos sobre los que (todavía) está basado el orden internacional. ${ }^{15}$ Siendo las coalitions of the willing una de las expresiones paradigmáticas —o bien, un instrumental fundamental - de esta concepción, presentan un gran reto para el desarrollo del derecho internacional.

Es bajo dicha coyuntura que las coalitions han adquirido una nueva importancia político-jurídica y empiezan a atraer la atención de la academia, tanto en los ámbitos del derecho y las relaciones internacionales, como en el económico. ${ }^{16}$ No obstante, sus diversas manifestaciones van mucho más allá de las coaliciones militares y no está nada claro si representan necesariamente instrumentos de política exterior estadounidense. De hecho, su proliferación se enmarca en un contexto mucho más complejo.

pean Union, A Secure Europe in a Better World, European Security Strategy, Bruselas, 12 de diciembre de 2003, pp. 9 y 10, en http://www.consilium.europa.eu).

15 Cabría preguntarse si ante estas concepciones discrepantes todavía podemos hablar de un orden internacional. Diversas y no siempre compatibles evoluciones, como la fragmentación y la concentración del derecho internacional ( $c f r$. Nolte, Georg, op. cit., nota 4), los intentos por reforzar las Naciones Unidas y la creciente importancia de mecanismos de gobernanza global al margen de las instituciones (global governance without a government), parecen sugerir que ya nos encontramos en un "(des)orden internacional" (Paulus, Andreas, "The War against...", cit., nota 2, en especial el apartado III. Competing Conceptions of the International Legal Order).

16 En el campo del estudio de las relaciones internacionales cabe destacar los siguientes artículos: Stewart, Patrick, "Beyond Coalitions of the Willing: Assessing US Multilateralism", Ethics and International Affairs, núm. 17, 2003; Tharoor, Shashi, "Why America still needs the United Nations", Foreign Affairs, septiembre-octubre de 2003; Stuart, D. T., "NATO and the Wider World: From Regional Collective Defence to Global Coalitions of the Willing", Australian Journal of International Affairs, núm. 58, 2004; Risse, Thomas, Effective Multilateralism..., cit., nota 13, así como Cooper, Andrew F., Stretching the Model of "Coalition of the Willing", The Centre for International Governance Innovation, Working Paper núm. 1, octubre de 2005, en http://www. cigionline.org. Desde la perspectiva jurídica, los análisis que versan directamente sobre el tema aún son escasos, aunque ya se cuenta con algunos artículos imprescindibles, como Byers, Michael, "Policing the High Seas: The Proliferation Security Initiative", American Journal of International Law, vol. 98, núm. 3, julio de 2004; así como el primer estudio sistemático sobre el tema por parte de: Benvenisti, Eyal, "Coalitions of the Willing" and the Evolution of Informal International Law, Tel Aviv University, Tel Aviv University Legal Working Paper Series, Law Faculty, Working Paper 31, enero de 2006, en http://law.bepress.com/taulwps/fp/art31. En cuanto a los estudios económicos, sobresale Drezner, Daniel W., Clubs, Neighborhoods and Universes: The Governance of Global Finance, en http://www.danieldrezer.com/research.html. 


\section{CONTEXTO}

La coalición militar contra Iraq no fue la primera, ni mucho menos la última coalition of the willing que ha aparecido en la escena internacional. Pero fuera de esta obvia constatación, es muy complicado trazar una genealogía, toda vez que aún no hay acuerdo sobre la extensión del concepto, $i$. e. sobre todo lo que éste realmente denota. De tal suerte, podemos hallar sus primeras manifestaciones a principios del siglo $\mathrm{XX}$, con la Comisión Electrotécnica Internacional de 1906 (CEI), ${ }^{17}$ o bien, en los años setenta, con el surgimiento de las redes intergubernamentales, como el Comité de Basilea para la Supervisión Bancaria de 1975. ${ }^{18}$

Más allá del origen, la imprecisión respecto a la extensión del concepto dificulta seriamente la identificación de los elementos que nos permiten hablar propiamente de una 'coalition of the willing', dando lugar a la enumeración de todo fenómeno parecido bajo este, a veces nuevo y a ratos viejo, rubro. Ello dependerá, principalmente, de los intereses académicos o políticos de quien use el término. Conforme al enfoque que reciba el tema, se pueden integrar al modelo desde organismos privados como la Agencia Mundial Antidopaje, ${ }^{19}$ pasando por organizaciones no gubernamentales (ONG) como la Asociación por el Trabajo Justo (FLA, por sus siglas en inglés), ${ }^{20}$ hasta organismos internacionales convencionales como la Organización para la Cooperación y el Desarrollo Econó$\operatorname{micos}(\mathrm{OCDE}) .{ }^{21}$

17 Véase infra III, 1.

18 Creado en 1975 por el G-10, el comité promueve la estabilidad financiera mediante recomendaciones sobre políticas bancarias, especialmente en materia de supervisión (auditorías y contabilidad) y detección de riesgos (alerta temprana de bancos débiles y crisis financieras). Sus miembros son los gobernadores de los bancos centrales de los trece países que participan en este esquema informal de coordinación política, carente de todo tipo de acto constitutivo (mayor información en www.bis.org).

19 Se trata de una fundación de derecho privado suizo ligada al Comité Olímpico Internacional, cuyo objeto social consiste en "promover y coordinar, a nivel internacional, la lucha contra el dopaje en el deporte" (artículo 4.1. del Acta Constitutiva de la Fundación, en http://www.wada-ama.org/en/dynamic.ch2?pageCategory.id=256). Para dicho propósito, emite estándares internacionales con gran repercusión en la formación de normas jurídicas.

20 Véase infra III, 1.

21 Como es conocido, la organización encabezada actualmente por José Ángel Gurría es de carácter selectivo, pues cuenta con una membresía de 30 países y con un procedimiento de acceso dificultado, diferente a la mayoría de las organizaciones 
Sin duda, todos estos organismos comparten ciertas características, ¿pero realmente nos permiten ubicarlas dentro del fenómeno que nos ocupa? ¿No estamos ampliando sus alcances al grado de confundirlo con cuestiones relacionadas mas no idénticas, como la gobernanza global y los regímenes autónomos (self-contained regimes) $?^{22}$

El peligro de incurrir en dicha confusión es grande a la luz de la proliferación de nuevos esquemas de acción internacional $-\mathrm{y}$ multilateral en específico-, en cuyo contexto se enmarcan las coalitions of the willing. Considero que uno de los principales y más interesantes retos para los iusinternacionalistas consiste actualmente en analizar detalladamente los vínculos entre todos estos mecanismos, así como explorar hasta qué punto es posible y conveniente construir un discurso jurídico coherente, capaz de abarcarlos sistemáticamente.

Precisamente en esa dirección se dirigen los argumentos de Paul S. Berman en torno a lo que él denomina "derecho y globalización". ${ }^{23}$ Sin

internacionales ( $c f r$. artículo XVI de la Convención de la OCDE, así como el documento Becoming a member of the OECD: the Accession Process, en http://www.oecd.org/ home/; la versión en español de la convención puede consultarse en el Diario Oficial de la Federación del 5 de junio de 1994). Pero, al mismo tiempo, sus recomendaciones, estudios y mecanismos de examen y presión tienen repercusiones inmediatas sobre las economías de Estados no parte. Ello, aunado a la gran importancia de esta organización en la construcción de la arquitectura financiera internacional, ha motivado a ciertos autores a calificarla como "club internacional", categoría dentro de la cual también suelen agruparse coaliciones ya típicas, como el Grupo de Acción Financiera (GAFI) (cfr. Drezner, Daniel W., Clubs, Neighborhoods and Universes..., cit., nota 16; Drezner se basa, a su vez en Walzer, Michael, Spheres of Justice, Nueva York, Basic Books, 1983).

22 Me refiero a los subsistemas de derecho internacional que exponen ciertas características autonómas, dando lugar al debate sobre la denominada "fragmentación del derecho internacional". Ésta se refiere, precisamente, a los problemas de coherencia normativa del orden jurídico internacional derivados de la proliferación de regímenes especiales, como el derecho de la Organización Mundial de Comercio, el derecho internacional ambiental o el de derechos humanos (colisiones entre subsistemas normativos). Comparto la conclusión de la Comisión de Derecho Internacional, en el sentido de que el término "regímenes autónomos" es incorrecto, toda vez que no existen sistemas internacionales "herméticamente aislados del derecho [internacional] general" ( $c f r$. Koskenniemi, Martti, "Dificultades derivadas de la diversificación y expansión del derecho internacional", Informe del Grupo de Estudio de la Comisión de Derecho Internacional, A/CN.4/L.682, CDI Quincuagésimo Octavo Periodo de Sesiones, 13 de abril de 2006, párr. 193).

23 Cfr. Berman, Paul Schiff, "From International Law to Law and Globalization, Columbia Journal of Transnational Law, núm. 43, 2005. 
embargo, Berman, además de abarcar prácticamente todo aquello que tiene que ver con la globalización, aboga por un discurso interdisciplinario excesivamente flexible, que tiende hacia una fusión del derecho y las relaciones internacionales, cuyos alcances son demasiado inciertos y no del todo inofensivos para los cánones sobre los que está basada la ciencia del derecho. ${ }^{24}$ Más apropiados me parecen, en cambio, los esfuerzos de la incipiente escuela del derecho administrativo global por crear un marco jurídico-conceptual, que pueda detectar nuevas y a veces difusas formas de regulación global, desarrollando al mismo tiempo principios y mecanismos para encauzarlas en un régimen de transparencia, revisión y responsabilidad, es decir, de legalidad. ${ }^{25}$

Estas líneas no tienen dicho alcance, simplemente pretenden llegar a una mejor comprensión del fenómeno específico. Un análisis sobre su significado tiene que empezar por la indagación de lo que el concepto 'coalition of the willing' designa. Finalmente, la determinación de su alcance (extensión), sin conocer bien a bien sus propiedades (intención), es un esfuerzo poco fructífero. Para dicho propósito, me serviré de las características comunes que presentan las coaliciones estudiadas por otros autore ${ }^{26} \mathrm{y}$ algunas otras de reciente surgimiento, o bien, que no han recibido mucha atención. Asimismo, me basaré en trabajos que, sin versar directamente sobre el tema, han desarrollado esquemas muy útiles para la identificación de sus propiedades, particularmente en el ámbito del mencionado derecho administrativo global. ${ }^{27}$

24 Sobre los riesgos de este tipo de aproximaciones, véase Koskenniemi, Martti, "Carl Schmitt, Hans Morgenthau, and the Image of International Law in International Relations", en Byers, Michael (ed.), The Role of Law in International Politics, Essays in International Relations and International Law, Oxford, OxfordUniversity Press, 2000, pp. 17-34.

25 Para saber más sobre esta corriente surgida en la Universidad de Nueva York, consúltese el número dedicado al tema de: European Journal of International Law, vol. 17, núm. 1, 2006.

26 Especialmente útil resulta la tipología propuesta por Benvenisti. Aunque las "características clave" que distingue se refieren directamente a los tipos - representando así un catálogo ordenado de las diversas denotaciones del concepto-, al mismo tiempo arrojan mucha luz sobre los elementos definitorios del concepto ( $c f r$. Benvenisti, Eyal, "Coalitions of the Willing"..., cit., nota 16).

27 Me refiero especialmente a Kingsbury, Benedict et al., "The Emergence of Global Administrative Law", Law \& Contemporary Problems, núm. 68, verano-otoño de 2005 . 


\section{PROPIEDADES}

\section{1. ¿Alianzas de "Estados afines" o agrupaciones ad hoc?}

Como su nombre lo indica, las coalitions of the willing son agrupaciones, aunque en muchos casos sumamente informales. ¿Pero qué tipo de elementos reúnen? Una de las caracterizaciones más comunes es que se trata de alianzas o coordinaciones de esfuerzos de "Estados afines" (like-minded states). ${ }^{28}$

Dicha presunción se debe, principalmente, al lenguaje utilizado por el agente que tiene la mayor capacidad de reunir a los demás participantes en una coalición determinada. ${ }^{29}$ Es en el ámbito de la lucha contra el terrorismo y, particularmente, en el combate contra la proliferación de armas de destrucción masiva (ADM), en donde el gobierno estadounidense se refiere con mayor frecuencia a los países afines: "To counter proliferation networks, we are working in common cause with like-minded states prepared to make maximum use of their laws and capabilities to deny rogue states, terrorists, and black marketeers access to WMD-related materials and delivery means". ${ }^{30}$ Y ello no debe sorprender, pues se trata de un rubro en donde la cooperación internacional es imprescindible, incluso —o quizá sobre todo- para la superpotencia. Es precisamente en este marco que el concepto 'multilateralism à la carte' cobra toda su expresión: "The challenges and opportunities before us are num-

28 Así, en un principio, Benvenisti en el multicitado artículo: Benvenisti, Eyal, "Coalitions of the Willing"..., cit., nota 16. En el mismo sentido: Byers, Michael, "Policing the High Seas...", cit., nota 16, pp. 543 y 544. Dicho sea de paso, una coordinación de esfuerzos de Estados implica, necesariamente, una agrupación de éstos bajo cualquier esquema, formal o informal.

29 Sobre la importancia del lenguaje oficial estadounidense en la percepción del fenómeno y su conceptualización, véase Cooper, Andrew F., Strechting the Model..., cit., nota 16 , pp. $2-4$.

30 The White House, President's Statement on the Proliferation Security Initiative, Press Release, Office of the Press Secretary, 31 de mayo de 2005, en http://www. whitehouse.gov/news/releases/2005/05/20050531-1.html. Énfasis añadido. Véase también National Strategy to Combat Weapons of Mass Destruction, diciembre de 2002, p. 6 en http://www.whitehouse.gov/nsc/. Sobre la lucha contra el terrorismo, en términos generales, véanse, por ejemplo, las declaraciones recientes del director de Inteligencia Nacional, John Negroponte: Department of State, USINFO, Like-Minded States must Work together to Thwart Terrorist Agenda, 24 de abril de 2006, en http://usinfo. state.gov. 
bing in their complexity and global in scope. We will succeed in meeting them only if we enlist the cooperation of other, like-minded states. So we must be also a resourceful sheriff, skilled in creating coalitions". ${ }^{31}$

Los peligros globales, entre ellos especialmente el terrorismo pero también las pandemias, los riesgos medioambientales y otros tantos más, requieren de la unión de esfuerzos del grueso de la comunidad internacional. EUA no ignora, de ninguna manera, esta realidad. Sin embargo, no se siente $-\mathrm{y}$, de hecho, no está - atado a las vías instituidas, que pueden ser útiles o no. Ante la gravedad y el carácter sumamente dinámico de dichos peligros, no es de esperarse que el Estado con la mayor capacidad de tomar la iniciativa internacional deje de hacerlo si aquéllas no responden rápidamente o de conformidad al resultado deseado: "Existing international institutions have a role to play, but in many cases coalitions of the willing may be able to respond more quickly and creatively, at least at the short term". ${ }^{32}$

Con ello hemos llegado a una de las características más evidentes de las coalitions: representan vías alternativas a las institucionales, concretamente a las organizaciones internacionales. Está aún por verse si son una amenaza para éstas o un complemento. Por lo pronto, observamos como el "Estado afín" no es únicamente el que comparte los fines sino, y ante todo, el que acepta los medios. Desde esta perspectiva, la afinidad se refiere, en primera línea, a la disposición de seguir el camino sugerido por el actor global más importante. La proximidad de valores y objetivos juega un papel, no hay duda, pero cae en segundo plano.

Las coaliciones militares ad hoc son un claro ejemplo de lo anterior, pero también lo son aquellas que, apartándose de acciones bélicas, se enmarcan dentro de la estrategia de seguridad nacional estadounidense. ${ }^{33}$

31 Haass, Richard N., From Reluctant to Resolute..., cit., nota 9. El énfasis es mío.

32 The National Security Strategy of the United States of America, marzo de 2006, p. 48, en http://www.whitehouse.gov/nsc/nss/2006/. Es interesante observar la evolución respecto a la concepción del papel de las coalitions frente a los organismos internacionales. Todavía la Estrategia de Seguridad Nacional 2002 se refiere a ellas como un refuerzo adicional, más que alternativo, a las organizaciones existentes: "The United States is committed to lasting institutions like the United Nations... as well as other long-standing alliances. Coalitions of the willing can augment these permanent institutions" (The National Security..., cit., nota 11, septiembre de 2002, p. VI).

33 Me refiero al conjunto de iniciativas y políticas de la administración Bush tendentes mantener la seguridad nacional, no a un documento en específico. 
En dicho marco destaca la coalición a la que se refiere el presidente Bush en el pasaje arriba citado, relativo a la proliferación de ADM: la Proliferation Security Initiative (PSI). ${ }^{34}$

Se trata de una iniciativa ya insinuada en la Estrategia Nacional para el Combate de ADM de 2002, ${ }^{35}$ y presentada por George W. Bush en la cumbre del G-8 en Cracovia, en mayo de 2003. Inicialmente contó con los Estados integrantes del G-8 y otros siete, el denominado núcleo, ${ }^{36}$ y actualmente reúne alrededor de setenta países. ${ }^{37}$ La PSI se dedica a coordinar esfuerzos entre las autoridades competentes de los países participantes para combatir el tráfico marítimo, terrestre y aéreo de ADM. Principalmente, comprende mecanismos de intercambio de información entre las agencias nacionales, la armonización de las legislaciones domésticas con el fin de reforzar las medidas de seguridad en fronteras, puertos y aeropuertos, y la adopción de una serie de principios sobre la detención, inspección y confiscación de medios de transporte, provenientes de Estados y actores no-estatales considerados, por los participantes de la PSI, sospechosos de traficar con ADM y materiales de uso dual. ${ }^{38}$ Asimismo, en el marco de la iniciativa se negocian una serie de tratados bilaterales para inspeccionar buques sospechosos en alta mar. ${ }^{39}$

34 La PSI es una de las coalitions of the willing mejor estudiadas a la fecha, sobresaliendo el trabajo de Michael Byers: Byers, Michael, "Policing the High Seas...", cit., nota 16. Benvenisti realiza un breve y puntual análisis: Benvenisti, Eyal, "Coalitions of the Willing”..., cit., nota 16. Véase también Spring, Baker, Harnessing the Power of Nations for Arms Control: The Proliferation Security Initiative and Coalitions of the Willing, The Heritage Foundation, 18 de marzo de 2004, en www.heritage.org.

35 National Strategy..., op. cit., nota 30, p. 6.

36 Éstos son Alemania, Canadá, Francia, EUA, Italia, Japón, Reino Unido y Rusia (G-8), así como Australia, España, Noruega, Países Bajos, Polonia, Portugal y Singapur.

37 Al no contar ni siquiera con su propia página $W e b$, tenemos que remitirnos a lo dicho por el presidente Bush y otros funcionarios de su gobierno ( $c f r$. The White House, President's Statement on the Proliferation Security Initiative, Press Release, Office of the Press Secretary, 23 de junio de 2006, en http://www.state.gov/t/isn/68267.htm; así como Joseph, Robert G., Broadening and Deepening our Proliferation Security Initiative Cooperation, en http://www.state.gov./t/us/rm/68269.htm).

38 Estas medidas a seguir se encuentran en los denominados Interdiction Principles for the PSI, que pueden consultarse en la página Web del Departamento de Estado estadounidense, página que contiene la información relevante sobre esta coalición http://www.state.gov/t/isn/c10390.htm.

39 A la fecha, se han celebrado acuerdos de este tipo entre EUA, por un lado, y Belice, Chipre, Croacia, Islas Marshall, Liberia y Panamá, por el otro. Se trata de 
Es justo mencionar que este mecanismo intergubernamental con pretensiones universales ${ }^{40}$ ha logrado combinar el respeto general al derecho internacional con incuestionables ventajas prácticas, derivadas directamente de su flexibilidad en la toma de decisiones. Ello podría sugerir que coalitions of the willing como la PSI brindan la oportunidad de contener al hegemón dentro de los cauces generales de la cooperación internacional, incluso en la persecución de sus intereses más fundamentales. La cuestión de si ello es razón suficiente para apoyar esta expresión particular del multilateralism á la carte es parte de otro debate. ${ }^{41}$ Empero, todo lo anterior no es óbice para constatar lo siguiente: es un proyecto intrínsicamente estadounidense, moldeado a sus formas de hacer política y que se circunscribe en toda una estrategia para avanzar rápida y eficazmente en la lucha contra el terrorismo y los "Estados villanos" (rogue States) con pretensiones nucleares (especialmente Corea del Norte e Irán), sin tener que sujetarse a las formas, tiempos y concesiones ${ }^{42}$ que demandan las instituciones internacionales.

Parte de dicha estrategia son otras iniciativas diseñadas en Washington, como la Container Security Initiative (CSI) ${ }^{43}$ y Megaports, ${ }^{44}$ así

acuerdos modelo propuestos por EUA, prácticamente idénticos ( $f f r$. http://www.state. gov/t/isn/c10390.htm).

40 Cfr. Joseph, Robert G., Broadening and Deepening..., cit., nota 37.

41 En este sentido: Byers, Michael, "Policing the High Seas...", cit., nota 16, p. 545.

42 'By sidestepping the 'least-common-denominator' approach for establishing international non-proliferation policy that is inherent in the consensus-based decision-making process of an international treaty regime, the PSI has already demonstrated that it will make a powerful contribution toward stemming proliferation" (Spring, Baker, op. cit., nota 34).

43 CSI es una iniciativa del Servicio de Aduanas y Protección Fronteriza (U.S. Customs and Border Protection) del Departamento de Seguridad Interior de EUA (Department of Homeland Security), cuya misión es colocar agentes aduaneros y migratorios de EUA en puertos extranjeros, con el fin de que revisen in situ los contenedores con destino a puertos estadounidenses. A cambio, EUA ofrece la misma posibilidad para los agentes de países participantes. CSI también elabora criterios para la detección de contenedores de alto riesgo. En CSI participan 26 Estados, de los cuales sólo Canadá y Japón han estacionado agentes en puertos estadounidenses. México no se ha incorporado a esta iniciativa. Mayor información en http://www.cbp.gov/xp/cgov/border_security/international_activities/csi/.

44 Mediante este esquema, la Administración Nacional de Seguridad Nuclear de EUA (National Nuclear Security Administration) provee a otros países de equipos de seguridad para sus puertos, destinados a la detección de armas nucleares y materiales radioactivos, que pueden ser utilizados para la construcción de "bombas sucias". Asimismo, entrena al personal del Estado respectivo en el uso de este equipo y otros 
como Cooperative Threat Reduction (CTR) ${ }^{45}$ Qué tan parecidas son todas ellas, lo demuestra la nueva propuesta lanzada el pasado 15 de julio, en el marco del G-8 celebrado en San Petersburgo, por los presidentes Bush y Putin: la Iniciativa Global para el Combate al Terrorismo Nuclear (GICNT, por sus siglas en inglés). ${ }^{46}$ GICNT está destinada a convertirse en la "coalición de coaliciones" en materia de combate al terrorismo nuclear, pues no sólo está planeado que incorpore a todos y cada uno de los programas comentados, sino también que emita guías prácticas para autoridades nacionales en materia de prevención, así como parámetros para la elaboración de leyes nacionales antiterroristas. ${ }^{47}$ Por si ello fuera poco, no se descarta que la GICNT llegue a constituir "la plataforma necesaria"48 para la implementación de la recientemente adoptada Convención Internacional para la Supresión de Actos de Terrorismo Nuclear. ${ }^{49}$

Los setenta y tantos países que participan en la PSI seguramente comparten su objetivo por convicción propia, así como muchos otros más que todavía no se han unido a ésta u otra iniciativa relacionada. Los

controles relacionados. A cambio exige el intercambio de la información recabada mediante el uso de los equipos mencionados. Actualmente, Megaports opera en Bahamas, España, Países Bajos, Singapur y Sri Lanka; también se ha iniciado su instrumentación en otros trece países; México no está dentro de ellos (véase $h t t p: / / w w w$. nnsa.doe.gov/megaports_initiative.htm).

45 Aquí muy difícilmente podemos hablar de una coalition of the willing, ya que CTR se limita a apoyar la construcción de una infraestructura eficiente para el control y desmantelamiento de armas nucleares en las antiguas repúblicas soviéticas, inclusive Rusia. Sin embargo, como el propio Departamento de Defensa menciona, no se trata de un programa de asistencia sino de una estrategia de defensa nacional (cfr. http://www. defenselink.mil/pubs/ctr/).

46 The White House, Office of the Press Secretary, Joint Statement by U.S. President George Bush and Russian Federation President V.V. Putin, Announcing the Global Initiative to Combat Terrorism, Press Release, 15 de julio de 2006, en http://www.white house.gov/news/releases/2006/07/20060715-2.html.

47 Cfr. The White House, Office of the Press Secretary, The Global Initiative to Combat Nuclear Terrorism, Fact Sheet, 15 de julio de 2006, en www.state.gov/t/isn/ $\mathrm{rls} / \mathrm{fs} / 69062 . \mathrm{htm}$.

48 Joseph, Robert G., The Global Initiative to Combat Nuclear Terrorism: A Comprehensive Approach to Today's Most Serious National Security Threat, Remarks to the Capitol Hill Club, 18 de julio de 2006, en www.state.gov/t/us/rm/69124.htm.

49 Dicha convención, en cuyas negociaciones nuestra cancillería tuvo un papel destacado, fue firmada por México el 12 de enero de 2006 ( $c f r$. Secretaría de Relaciones Exteriores, Comunicado de Prensa núm. 009, 12 de enero de 2006, en http://www.sre. gob.mx/comunicados/comunicados/2006/enero/b_009.htm). 
esquemas y métodos que comprende todo este andamiaje contra el terrorismo y la proliferación de $\mathrm{ADM}$, no obstante, provienen de una manera particular de entender la cooperación multilateral, forjada por la administración Bush. A esta concepción, los denominados países "afines" se alinean, ya sea por los incentivos involucrados, como los apoyos al desarrollo de infraestructuras de seguridad y desarme, o bien, simplemente por no quedarse fuera del grupo de naciones dispuestas a luchar contra las peores amenazas que vive por ahora la humanidad, con todo lo que ello implica. Sólo algunos pocos, sobre todo los miembros del G-8, tienen el privilegio de integrar el núcleo en ésta o aquella formación particular, acercándose así un poco a la auténtica noción de 'afinidad', que, por definición, es poco compatible con la idea de un sheriff que reúne a sus destacamentos, según lo demande el asunto en cuestión. ${ }^{50}$

Ahora bien, habíamos mencionado que reducir las coalitions of the willing a instrumentos de política exterior estadounidense es probablemente falso y, sin duda, miope. Nadie duda sinceramente que lo sean, pero no lo son exclusivamente. En dicho sentido, es necesario ir más allá del discurso oficial estadounidense para ver cuáles son sus criterios básicos, no específicos, de pertenencia.

Dentro de los ejemplos hasta el momento proporcionados podemos constatar que los Estados no son los únicos participantes posibles. La CEI, e. g., es una asociación de derecho privado suizo, ${ }^{51}$ cuya función consiste en establecer estándares normativos mundiales en materia de tecnología eléctrica y electrónica. Su composición es mixta, pues sus miembros de pleno derecho son los órganos nacionales encargados de la normalización en materia eléctrica y electrotécnica, o bien, en cuestiones de normalización en general. Es cierto que la mayoría de ellas son asociaciones privadas, pero algunas pertenecen al sector público, ${ }^{52}$ en tanto que otras tienen una estructura mixta. ${ }^{53}$ En este mismo rubro, la Organización Internacional de Normalización, mejor conocida como ISO, ocupa un lu-

50 Paráfrasis de Haass, Richard N., From Reluctant to Resolute..., cit., nota 9.

51 Cfr. IEC Statutes and Rules of Procedure, 2001; en http://www.iec.ch/tiss/directi ves.htm.

52 Como es el caso de México, que está representado por la Secretaría de Economía mediante la Dirección General de Normas.

53 La Asociación Española de Normalización y Certificación fue designada por el Ministerio de Industria y Energía, y sus funciones están determinadas por Decreto Real; su acto constitutivo es un estatuto de derecho privado (cfr. http://www.aenor.es). 
gar muy especial. ISO no es una organización internacional tradicional, basada en un tratado entre Estados, sino una ONG cuya composición es prácticamente idéntica a la CEI, ${ }^{54}$ institución de la cual surgió. Su finalidad es, como bien se sabe, establecer estándares mundiales para normas técnicas que versan sobre la calidad de productos y servicios.

Existen también algunas coaliciones de naturaleza enteramente privada, como la FLA. Aquí estamos ante la presencia de una iniciativa creada por la administración Clinton, ${ }^{55}$ que lucha por la erradicación de formas injustas de trabajo, como el de menores de edad en la manufactura. Se define como una "coalición de intereses múltiples" (multi-stakeholder coalition) y comprende a varias ONG, empresas líderes en el sector manufacturero y 194 universidades estadounidenses. La FLA ha fijado, mediante el Código de Conducta en el Lugar de Trabajo y los Puntos de Referencia para observar su cumplimiento, ${ }^{56}$ una serie de estándares mínimos en materia de justicia laboral, que reflejan derechos básicos, como los que reconoce la mayoría de las legislaciones nacionales y las convenciones de la Organización Internacional del Trabajo, pero que en múltiples ocasiones no son respetados por empresas transnacionales, ni se hacen respetar por las autoridades locales. Asimismo, la FLA se ha convertido en un importante mecanismo de monitoreo en las empresas involucradas, con base en el principio "nombrar y apenar" (naming and shaming).

Dentro de este tipo de coaliciones, Benvenisti se refiere a un par de organismos de carácter financiero, que fijan estándares internacionales en materias como títulos y garantías de crédito. ${ }^{57}$ Aquí la participación de los Estados es prácticamente nula, ${ }^{58}$ pero las repercusiones de sus decisiones sobre los derechos bancarios nacionales son significativas. Una

54 México está igualmente representado por la Dirección General de Normas de la Secretaría de Economía. Mayor información puede encontrarse en www.iso.org.

55 Cfr. Benvenisti, Eyal, "Coalitions of the willing”..., cit., nota 16.

56 Véase $h t t p: / / w w w$ fairlabor.org/all/code/ y http://www.fairlabor.org/docs/monitor ingsp.doc.

57 Benvenisti se refiere a la Unión Internacional de Seguros de Crédito e Inversión (Unión de Berna) y a la Comisión sobre Técnicas y Prácticas Bancarias ( $c f r$. Benvenisti, Eyal, "Coalitions of the Willing”..., cit., nota 16).

58 La mayoría de las instituciones integrantes de la Unión de Berna son sociedades mercantiles. De hecho, no se trata de una representación por Estado, pues existen diferentes miembros provenientes del mismo país, como en el caso de EUA. Dentro de los miembros se encuentra Bancomext (cfr. http://www.berneunion.org.uk/home.htm). 
de ellas, la Comisión sobre Técnicas y Prácticas Bancarias, pertenece a la Cámara de Comercio Internacional (CCI), organismo que merece mayor análisis a la luz de la propagación de las coalitions of the willing. Se trata de la "organización mundial de las empresas", según su propia frase publicitaria, pero desarrolla, mediante la elaboración de códigos de conducta, reglas no vinculantes, contratos modelo y posicionamientos "políticos", inter alia, especies de políticas públicas en diversas materias, que van desde la bancaria hasta la fiscal y la propiedad intelectual. ${ }^{59}$ Ello rebasa una evolución normal de la lex mercatoria y merece confrontarse a la evaluación de los publicistas del derecho internacional. ${ }^{60}$

A la luz de estos ejemplos, queda claro que los integrantes de las coalitions of the willing no son solamente de carácter estatal, por lo que no podemos hablar más de agrupaciones de "Estados afines", sino de 'agrupaciones de actores reunidos sobre una base ad hoc' o, simplemente, de 'agrupaciones ad hoc'. ${ }^{61}$ Incluso en el ámbito de las coaliciones dedicadas a la lucha contra el terrorismo internacional y la proliferación de

59 Toda esta información puede consultarse en http://www.iccwbo.org/.

60 Este asunto ha sido estudiado desde la perspectiva de la fragmentación del derecho internacional, especialmente por: Fischer-Lescano, Andreas y Teubner, Gunther, Regime-Kollisionen, Zur Fragmentierung des globalen Rechts, Francfort del Meno, Suhrkamp Verlag, 2006, pp. 99-110). Asimismo, pero quizá desde un punto de vista más práctico para el análisis de las coalitions of the willing, el derecho administrativo global ha empezado a prestarle atención (Kingsbury, Benedict et al., op. cit., nota 27, p. 29).

61 Como ya se mencionó, la 'afinidad' como propiedad de las coalitions es, en algunos casos, problemática. Y ello no es exclusivo de las coaliciones creadas por EUA. Incluso en casos aparentemente inofensivos, como la estandarización de normas técnicas, la fuerza de atracción de los organismos respectivos radica más en la adaptación que en la proximidad de ideas. Es cierto que en muchos casos no tiene sentido ir en contra de la corriente, pero en muchos otros ésta es simplemente demasiado fuerte, dejando a los actores menos poderosos pocas alternativas de elección (Daniel Drezner explica la fuerza de atracción de los "clubes" de la arquitectura financiera internacional sobre las economías en desarrollo, así como la asimétrica relación costo-beneficio para éstas al adoptar ciertos estándares y medidas desarrollados por aquellos: Drezner, Daniel W., Clubs; Neighborhoods and Universes..., cit., nota 16). En algunos casos determinados, como la CCI, probablemente sí se pueda hablar propiamente de 'actores afines'; en otros, sobre todo los que emanan de los G-8 y G-10, existen 'núcleos de actores afines', que atraen a los demás participantes con base en mecanismos de premios y castigos. En común tienen todos estos tipos el carácter ad hoc: las coalitions reúnen a diferentes actores, no necesariamente afines, según dicte la causa. Por dichas razones, considero pertinente eliminar el adjetivo 'afin' de los elementos definitorios de las coalitions y hablar simplemente de actores reunidos sobre una base ad hoc. 
ADM, cuestiones que se enmarcan en las funciones coactivas del Estado, la tendencia es empezar a incorporar al sector privado, como lo muestran los planes que existen respecto a la GICNT. ${ }^{62}$

Si dicha caracterización es correcta, cabría preguntarse si las coalitions también comprenden aquellas redes civiles que, motivadas por las más diferentes y nobles causas, operan a escala mundial. Andrew Cooper aboga por esta "extensión del modelo coalition of the willing", ${ }^{63}$ misma que parece, prima faciae, compatible con los elementos hasta ahora identificados.

En efecto, las coalitions pueden llegar a configurar amplias redes, agrupando a gobiernos nacionales, ONG, empresas y hasta al sector académico. No hay límites al respecto, pues, como vimos, se trata de aglomeraciones de actores en términos generales y según dicte la causa, es decir, ad hoc. Sin embargo, lo anterior no significa que las coaliciones sean, necesariamente, redes transnacionales.

Si entendemos el concepto 'red transnacional' latu sensu, i. e. como coordinación de esfuerzos de actores internacionales con un fin determinado, podría aplicar el principio: toda coalición es una red transnacional pero no toda red transnacional es una coalición. Hablaríamos, entonces, de redes transnacionales, más ciertos elementos. Así, redes civiles que operan a escala mundial, como la Coalición por la Corte Penal Internacional y la Campaña Internacional para la Prohibición de las Minas, o bien, redes de intereses múltiples como el Foro Global de Investigación Agropecuaria (GFAR, por sus siglas en inglés), ${ }^{64}$ podrían incorporarse al modelo, en el sentido propuesto por Cooper. ${ }^{65}$

62 "There is also a large role for the private sector to play mitigating the risk of nuclear terrorism. In the United States as in other countries, a substantial portion of the nuclear infrastructure is controlled by private sector utilities, laboratories, or university research centers or institutes. By working closely with these private entities, as well as those that supply and insure them, we can stimulate the development of best practices, risk management approaches, and codes of conduct" (Joseph, Robert G., The Global Initiative..., cit., nota 48).

63 Cfr. Cooper, Andrew F., Stretching the Model..., cit., nota 16.

64 GFAR es, al igual que la FLA, una ONG de intereses múltiples, cuyo objetivo principal es apoyar la investigación agropecuaria, especialmente en países en vías de desarrollo, con el fin de fortalecer la lucha contra la pobreza. Más información en http://www.egfar.org.

65 Cfr. Cooper, Andrew F., Stretching the Model..., cit., nota 16, pp. 5 y ss. 
Ahora bien, otra propiedad que ha sido recurrente en nuestros ejemplos es la creación de la coalición como vía alternativa a los foros internacionales establecidos. A estas alturas, podemos sostener, sin temor a equívocos, que estamos ante la presencia de una forma alterna no sólo de entender el multilateralismo sino de practicarlo. Ello implica un desafío más que un apoyo decidido a los procesos formales internacionales, como la celebración y ratificación de tratados, en este caso la Convención de Ottawa y el Estatuto de Roma. Y aunque ello podría calificarse como un argumento débil, ¿qué tanto le puede convenir a estas ONG entrar dentro de un catálogo de agrupaciones desafiantes de los procesos y las instituciones que apoyan?

Un argumento fuerte a favor de la delimitación de las coalitions respecto a las redes civiles transnacionales estriba en que éstas carecen de otra característica que ha sido constante en todos los demás casos: la elaboración de estándares normativos internacionales. Sin contar con tal elemento, me parece que la extensión del 'modelo coalition of the willing' es forzada, convirtiendo al concepto en una etiqueta para toda aglomeración derivada de la globalización.

La elaboración de estándares normativos, por su parte, significa una fuerte incidencia en el derecho, y no sólo internacional, como lo muestran las coaliciones de carácter técnico y financiero. A esta doble faceta, es decir, la aparente indiferencia hacia las formas jurídicas y la fuerte incidencia en sus conceptos fundamentales, como la creación y ejecución normativas, le denomino 'el carácter amorfo de las coalitions of the willing', que, a mi juicio, constituye su propiedad esencial.

\section{Agrupaciones amorfas}

Las coalitions carecen de una forma jurídica regular, siendo en algunos casos personas de derecho privado (CCI, CEI, ISO, FLA, e. g.) y prescindiendo en otros de toda clase de personalidad jurídica (PSI y Comité de Basilea, entre otros). La falta de formalidad jurídica no se agota en su constitución, se extiende a sus funciones. Las coalitions no crean normas jurídicas y, aparentemente, no pretenden hacerlo: "These partnerships... rely on voluntary adherence rather than binding treaties. They are oriented towards action and results rather than legislations or rule-making". ${ }^{66}$ Hasta cierto punto ésto es obvio, toda vez que no se trata de ór- 
ganos facultados para la creación normativa. Incluso en el caso menos evidente de la costumbre internacional, tenemos que ser muy cautelosos, pues, al no expresar intención respecto a la generación de reglas vinculantes, es muy difícil extraer el convencimiento jurídico sobre las prácticas de los Estados que algunas de ellas generan. ${ }^{67}$ Por su parte, es justo subrayar que las coalitions no cometen hechos antijurídicos, al menos no sistemáticamente ni de cualquier otra manera que pueda atribuírsele como parte de sus características. En la mayoría de los casos se trata de "diferentes tipos de acción intergubernamental coordinada, que se valen de las competencias ya conferidas a los gobiernos nacionales por el derecho internacional". 68

Todo lo anterior nos podría llevar a la conclusión de que las coalitions of the willing no son un fenómeno jurídico, que su naturaleza es más bien política. En ese sentido, su estudio estaría mejor ubicado en la ciencia política, y los juristas no deberían angustiarse por la falta de forma regular, pues, finalmente, no es factible ni deseable que todo actor social esté determinado por el derecho. La cooperación política internacional siempre ha existido y es completamente normal y saludable que los diferentes gobiernos nacionales, incluso por medio de sus agencias especializadas, celebren memoranda de entendimiento o que simplemente se reúnan períodicamente para acordar acciones conjuntas, sin la celebración de instrumento jurídico alguno. Asimismo, no hay nada extraño en que los (ya no $\tan )$ nuevos actores internacionales se organicen para la persecución de diversos fines. Pero estos mecanismos intergubernamentales y organizaciones civiles o de intereses múltiples no son, per se, coalitions of the willing y, por tanto, no forman parte de lo que denominamos 'agrupación amorfa'. Lo amorfo se define en función de la forma y la coalition of the

67 Con ello no quiero decir que la introducción de "prácticas apropiadas" (cfr. Benvenisti, Eyal, “Coalitions of the Willing”..., cit., nota 16) no represente problema alguno para la vigencia de normas existentes. Este es el caso de la PSI respecto a las prácticas sobre el derecho de visita contemplado en el artículo 110 de la Convención de Naciones Unidas sobre el Derecho del Mar. Sin embargo, y como bien señala Michael Byers, no hay a la fecha indicios claros de que se pretenda generar una nueva costumbre internacional sobre la detención y confiscación de buques en alta mar, pues su cristalización, en virtud de la celebración de una serie de tratados bilaterales, se dificulta al estar éstos concebidos como excepciones a la costumbre establecida ( $c f r$. Byers, Michael, "Policing the High Seas...", cit., nota 16, pp. 532-536).

68 Benvenisti, Eyal, “Coalitions of the Willing”..., cit., nota 16. La traducción es mía. 
willing en función del derecho: ésta se puede describir como una agrupación sin forma regular que produce pautas de conducta no objetivas, pues genera una serie de reglas internacionales que no están basadas (o derivadas) en el artículo 38 del Estatuto de la Corte Internacional de Justicia, y que, sin ser vinculantes, son altamente eficaces. Las coalitions no son indiferentes hacia el derecho, todo lo contrario - Benvenisti habla incluso del "derecho de las coalitions of the willing"- ${ }^{69}$ pero desprecian el pensamiento formal sobre el que está basado, sustituyéndolo por un pensamiento orientado por fines políticos específicos. A esta manera irregular e intencionalmente confusa de incidir en la creación de normas internacionales es a lo que denomino amorfo.

Ahora bien, habíamos mencionado que las coalitions se enmarcan en todo un contexto de nuevas manifestaciones y acciones multilaterales. Con ellas comparten la incidencia en el sistema de fuentes del derecho internacional, pues todas consisten en mecanismos difusos de regulación internacional e incluso transnacional, afectando no sólo al orden jurídico internacional sino también a los derechos nacionales. El derecho administrativo global denomina este fenómeno "regulación administrativa globalizada", y ha identificado al menos cinco diferentes tipos, ${ }^{70}$ de los cuales tres corresponden a las coalitions of the willing; veámoslos:

a) la administración de organizaciones internacionales formales; b) la administración de redes intergubernamentales informales, i.e. la coordinación política entre agencias gubernamentales de diversos países; c) la administración formal de redes intergubernamentales mediante la celebración de tratados, acuerdos interinstitucionales u otros regímenes de cooperación; d) la administración de entes híbridos público-privados; y e) la administración de instituciones privadas.

No es difícil constatar que los tipos b), d) y e) coinciden con el fenómeno aquí analizado. En el tipo b) destacan el Comité de Basilea y la PSI; de la clase d) no hemos visto ejemplos en el presente trabajo, pero varios autores identifican aquí a la Internet Corporation for Assigned Names and Numbers (ICANN) ${ }^{71}$ o al Fondo Mundial de Lucha contra el

70 Cfr. Kingsbury, Benedict et al., op. cit., nota 27, pp. 20 у 21.

71 Cfr. Idem, p. 22. 
SIDA, la Tuberculosis y la Malaria; ${ }^{72}$ finalmente, la letra e) está perfectamente representada por CCI, CEI, FLA, ISO, la Unión de Berna y demás.

Aunque los cinco tipos comparten el mismo problema general, es posible diferenciar, pues a) y c) se manifiestan mediante formas de creación normativa que, si bien son problemáticas, pertenecen a viejas discusiones jurídicas. Finalmente, hablamos de actores bien definidos (organismos internacionales y Estados) que, probablemente, abusan de las herramientas jurídicas que les han sido conferidas. El tipo a) se refiere, por ejemplo, a las disputables facultades legislativas del CSNU, ${ }^{73}$ en tanto que c) a la denominada "disolución del Estado"74 como sujeto unitario de derecho internacional, en virtud de mecanismos de coordinación entre agencias estatales, $i$. e. entidades administrativas regulativas, de los diferentes Estados. ${ }^{75} \mathrm{El}$ primer caso es un problema de facultades ultra vires $\mathrm{y}$, más allá, de la excesiva concentración del derecho internacional. ${ }^{76} \mathrm{El}$ segundo presenta, inter alia, cuestionamientos de derecho interno, especialmente sobre la constitucionalidad de ciertos actos e instrumentos de política exterior, como los acuerdos interinstitucionales en nuestro país. Por otro lado, observamos como en los tipos b), d) y e) se trata de agrupaciones que carecen in toto de facultades regulatorias, operando al margen —no en contra- del derecho, por lo que no emiten normas jurídicas de ningún tipo, tampoco administrativo, sino estándares normativos, respaldados por la justicia o razón de la causa particular, sea la construcción de una arquitectura financiera más estable, el combate al terrorismo, la calidad de productos y servicios o condiciones más justas en los lugares de trabajo. A qué grado repercuten dichos estándares, también en México, lo ilustran las recomendaciones del Grupo de Acción Financiera (GAFI).

El GAFI ${ }^{77}$ fue creado en 1989 por el entonces G-7, con el afán de combatir el lavado de dinero, sobre todo ante la creciente repercusión de este

72 Cfr. Benvenisti, Eyal, “Coalitions of the Willing”..., cit., nota 16.

73 Cfr. Kingsbury, Benedict et al., op. cit, nota 27, p. 21.

74 Cfr. Slaughter, Anne-Marie, "Governing the global economy through government networks", en Byers, Michael, The Role of Law..., cit., nota 24, pp. 178 y 179.

75 Cfr. Kingsbury, Benedict et al., op. cit., nota 27, p. 21.

76 Al respecto véase Nolte, Georg, op. cit., nota 4.

77 Toda la información puede consultarse en http://www.fatf-gafi.org. Muy útil resultan también los análisis de Benvenisti y Drezner: Benvenisti, Eyal, "Coalitions of the Willing"..., cit., nota 16; Drezner, Daniel W., Clubs, Neighborhoods and Universes..., cit., nota 16 . 
fenómeno delictivo en el sistema financiero internacional. Este mecanismo de coordinación política (tipo b) reúne en la actualidad a 31 Estados y dos organismos regionales (la Unión Europea y el Consejo de Cooperación del Golfo). México se unió al GAFI en 2000, para lo cual no fue necesario acto jurídico alguno, toda vez que - como ya es casi obvioesta coalition no está basada en tratado, ni cuenta con acto constitutivo, estatuto o reglamento, i. e. no tiene status iuris. ${ }^{78}$ Como tal, tampoco tiene capacidad jurídica, por lo que se limita a emitir recomendaciones no vinculantes para combatir el lavado de dinero y, desde 2001, la financiación del terrorismo. Sus recomendaciones consisten en propuestas legislativas concretas para los Estados. En la primera materia ha emitido cuarenta, en la segunda nueve, los denominados "estándares GAFI". Para verificar el cumplimiento de dichas "sugerencias", GAFI ha elaborado un mecanismo de monitoreo consistente en reportes de los Estados sobre las medidas legislativas adoptadas (cuestionarios) y controles in situ llevados a cabo por grupos de expertos de otros gobiernos participantes. Si algún Estado no realiza las modificaciones legislativas o no lo hace de manera apropiada, los demás integrantes pueden ejercer presiones de carácter económico y, si es necesario, acordar la expulsión del Estado incumplido. ${ }^{79}$

Es curioso observar como dicha presión político-económica resulta, en muchos casos, más eficaz que la fuerza obligatoria de las normas contenidas en tratados, resoluciones de ciertos órganos o decisiones de tribunales internacionales. Conocemos en México el largo camino que tiene que atravesar frecuentemente un tratado para ser desarrollado legislativamente; la reticencia que persiste hacia las decisiones de los tribunales de los que México es parte no es todavía - y lamentablemente-cosa del pasado. Con las recomendaciones del GAFI el panorama es distinto.

Desde antes de su ingreso, México ha empezado a implementar las propuestas en materia de lavado de dinero. ${ }^{80}$ Mediante una amplia refor-

78 A diferencia de lo que muchas veces se piensa, GAFI no es parte de la OCDE, simplemente tiene sus oficinas en el edificio de la organización.

79 GAFI (FATF, por sus siglas en inglés), Methodology for Assesing Compliance with FATF 40 Recommendations and the FATF 9 Special Recomendations, 27 de febrero de 2004 (actualizada a junio de 2006), en http://www.fatf-gafi.org.

80 Así, por ejemplo, en 1996 se tipificó el delito de lavado de dinero en el artículo 400 bis del Código Penal federal, previamente contemplado como delito fiscal ( $c f r$. Cuisset, André, "La importancia para México de una política coherente y eficiente en 
ma a diversas leyes financieras, que entró en vigor en enero de $2004,{ }^{81}$ se dio un gran paso hacia la implementación completa de las mismas, así como también de las recomendaciones sobre financiación al terrorismo, aunque aquí todavía está pendiente la reforma al Código Penal Federal, presentada en septiembre de 2003 por el Ejecutivo federal al Senado. ${ }^{82}$ No quiero decir, de ninguna manera, que ello sea negativo, al contrario, me parece sumamente importante que México cuente con un marco jurídico adecuado para contrarrestar estos males. Lo único que me interesa subrayar es cómo estas importantes modificaciones legislativas reflejan el impacto que pueden llegar a tener las coalitions en los diferentes ordenamientos jurídicos nacionales; particularmente, cómo son capaces de reglamentar a nivel transnacional sin basarse en la noción de validez jurídica, sino en la justa razón de la causa y en el poder real que ostentan. En la explicación de la iniciativa presentada al Legislativo en septiembre de 2003, contenida en el Cuarto Informe de Gobierno del presidente Fox, se menciona que el objetivo de ésta es "dar cumplimiento a las recomendaciones del GAFI [para] evitar posibles medidas de éste contra México por falta de cooperación". ${ }^{83}$ La Secretaría de Hacienda y Crédito Público, por su parte, justifica la emisión de reglas de carácter general en la materia de un modo similar: "Es importante destacar que las recomendaciones del GAFI tienen una fuerza vinculativa toda vez que su adopción es voluntaria y dicho grupo concentra a los países de mayor relevancia financiera". ${ }^{84}$ Estas justificaciones oficiales reflejan claramente como "gobiernos afectados... cumplen con estas leyes no por una noción de de-

materia de lavado de dinero", en Roemer, Andrés y Buscaglia, Eduardo (comps.), Terrorismo y delincuencia organizada. Un enfoque de derecho y economía, México, UNAM, Instituto de Investigaciones Juridicas, 2006, p . 80.

81 Cfr. Diario Oficial de la Federación, 28 de enero de 2004.

82 Cfr. Senado de la República, LIX Legislatura, Gaceta Parlamentaria, año 2003, núm. 4, 11 de septiembre de 2003. Esta iniciativa incluye la importante introducción del tipo de terrorismo internacional a nuestra legislación penal federal.

83 Presidencia de la República, Cuarto Informe de Gobierno del C. presidente Vicente Fox Quesada, 1o. de septiembre de 2004, en http://cuarto.informe.presidencia. gob.mx/index.php?idseccion $=10$.

84 Manifestación de Impacto Regulatorio para el Anteproyecto de Resolución por la que se expiden las Disposiciones de Carácter General a que se refieren los artículos 115 de la Ley de Intituciones de Crédito y 124 de la Ley de Ahorro y Crédito Popular, en http://www.cofemermir.gob.mx/inc_lectura_regioncontentall_text.asp? submitid $=4478$. 
ber jurídico, sino más bien por el miedo a los efectos adversos del incumplimiento". ${ }^{85}$

El impacto legislativo transnacional de las recomendaciones del GAFI no se agota allí, pues dirige sus propuestas más allá de su membresía. Así, destaca Benvenisti, ${ }^{86}$ más de 130 países han adoptado las recomendaciones sobre lavado de dinero, convirtiéndose en el estándar internacional en la materia. Este club internacional con alcances jurídicos casi universales, logra dicho objetivo mediante la incorporación de los Estados no dispuestos a colaborar en la llamada lista de países y territorios no-cooperativos. ${ }^{87}$ Ello no representa únicamente un medio para evidenciar a los Estados conforme al principio de "nombrar y apenar", pues, en caso de no reaccionar rápidamente al llamado de atención, los Estados enlistados pueden hacerse acreedores de sanciones internacionales, pues GAFI puede recomendar a sus integrantes la adopción de contramedidas: "in cases where progress has stalled, the FATF will recommend the application of further countermeasures which should be gradual, proportionate and flexible regarding their means and taken in concerted action towards a common objective". ${ }^{88}$ Las contramedidas ${ }^{89}$ sugeridas consisten en sanciones económicas, que van desde la restricción hasta prohibición de transacciones financieras con los Estados no-cooperativos.

Me parece que GAFI da perfectamente cuenta del carácter amorfo de las coalitions of the willing: un mecanismo informal basado en el consenso político y la libre voluntad de los participantes que se atribuye la capacidad moral de recomendar la incorporación de estándares normativos en las legislaciones nacionales de todo el mundo. Para dicho propósito, parte de la presunción de una especie de validez universal de dichos estándares, parecida al discurso de los derechos humanos pero sin contar con la amplia aceptación de éste por la comunidad internacional. Y todo ello, so pena de recomendar la adopción y ejecución de medidas coercitivas, carentes de todo fundamento jurídico, pues simplemente no hay nor-

85 Benvenisti, Eyal, “Coalitions of the Willing”..., cit., nota 16. La traducción es mía.

86 Idem.

87 GAFI, Annual Review of Non-Cooperative Countries and Territories, 2005-2006, 23 de junio de 2006, en http://www.fatf-gafi.org.

88 Ibidem, párrafo 20.

89 En diciembre de 2001, GAFI decidió implementar contramedidas a Nauru. 
ma jurídica lesionada. ${ }^{90}$ Quizá dichas medidas sean de carácter político, sin pretensión legal alguna; puede ser, pero entonces ya no parece tener mucho sentido distinguir entre el derecho y la política internacionales.

\section{CONCLUSIONES}

"We have been here before", ${ }^{91}$ dice Martti Koskenniemi al explicar que la tendencia académica reciente ${ }^{92}$ de fusionar el estudio del derecho y las relaciones internacionales no es realmente tan novedosa, reflejando, en buena medida, la tensión entre positivismo jurídico y realismo político que se vivió respecto a la Constitución de la República de Weimar. Dicha corriente - hay que decirlo - corresponde al estado actual de las cosas. Sería poco sensato no admitir que la falta de equilibrio de poder en la comunidad internacional tambalea los cimientos jurídicos sobre los que descansa. Conceptos fundamentales como la igualdad jurídica de los Estados se interponen a menudo con las necesidades políticas de quienes ostentan los medios para actuar decididamente en la escena internacional. Ello conduce a dichos actores, particularmente EUA, a abandonar las vías instituidas cuando éstas no tienen la disposición o la capacidad

90 Caso distinto, pero no menos problemático, es la adopción y ejecución de sanciones por violaciones a normas jurídicas internacionales, como en caso de incumplimiento de resoluciones del CSNU. Aquí, las coalitions suelen atribuirse no sólo la facultad de ejecutar sanciones a falta de voluntad política del CSNU para emitirlas, sino también la competencia para determinar la existencia de las violaciones respectivas. Esta doble función - interpretativa y sancionadora de la ley sin base legal — fue evidente en la coalición militar de 2003 y podría repetirse, aunque no necesariamente como agrupación militar, contra Irán, por el eventual incumplimiento de la Resolución 1696 del CSNU. Al respecto, el representante permanente de EUA ante Naciones Unidas, John R. Bolton, ya ha anunciado la posible formación de una coalición independiente para adoptar contramedidas de carácter económico contra Irán, en caso de que el CSNU no sea capaz de hacerlo por los probables vetos de de Rusia y China (cfr. U.S. May Curb Iran, Los Angeles Times, 26 de agosto de 2006, en http://www.latimes.com/la-fg-iran 26aug26,0,5564225.story? track=mostviewed-sectionfront).

91 Koskenniemi, Martti, "Carl Schmitt, Hans Morgenthau...”, cit., nota 24, p. 17.

92 Anne-Marie Slaughter es una de las más conocidas representantes de esta escuela, dentro de la cual también podemos ubicar a Paul S. Berman, aunque éste va más allá en su enfoque interdisciplinario, particularmente por la inclusión de la escuela de la legal consciousness (cfr. Berman, Paul Schiff, op. cit., nota 23; Slaughter, Anne-Marie et al., "International Law and International Relations Theory: A New Generation of Interdisciplinary Scholarship", AJIL, vol. 92, núm. 3, 1998). 
de responder con eficacia ante los riesgos mundiales, cuyo dinamismo, no hay duda, plantea importantes desafíos a los tiempos y formas de la organización internacional vigente. Bajo esta coyuntura, las coalitions of the willing constituyen un medio idóneo para sustituir procesos e instituciones tradicionales, por lo que son, si bien no exclusivamente, instrumentos muy eficientes de política exterior estadounidense.

Pero el fenómeno no se agota allí. La creciente interacción de actores y la diversificación de temas globales también invitan a la creación de agrupaciones ad hoc, en donde la causa específica es principio y fin de su acción, $\mathrm{y}$, sobre todo, la fuente exclusiva de su legitimidad. El carácter ad hoc de las coalitions refiere directamente al discurso tecnocrático, conforme al cual la justa razón desplaza al concepto formal de validez como fuente de legitimidad internacional. El proceso - si es que hay tal- es flexible, pues la rigidez obstaculiza el buen manejo del asunto en cuestión. Y no sólo el proceso dificulta su operación, la constitución de una coalition puede convenir de tal o cual forma, o bien, de ninguna.

Al mismo tiempo, las coalitions aspiran a la mayor eficacia posible de sus estándares. Éstos tienen que ser reconocidos en los países civilizados, para su propio bien y el de la comunidad internacional en su conjunto. Para ello, las formas jurídicas siguen siendo imprescindibles, pero incorporarse a ellas sería contrario a su naturaleza. De tal suerte, tratan de incidir desde afuera, de una u otra manera, siempre difícil de determinar. Estándares se traducen en legislación nacional sin seguir el fastidioso camino de un tratado, con todo y lo que ello implica, como los procedimientos convalidatorios internos; medios de coacción moral se diluyen con conceptos jurídicos fundamentales, como el hecho ilícito y la sanción. No hay duda, su carácter amorfo trae consigo serios problemas de transparencia y responsabilidad, y, peor aún, borra la distinción fundamental entre tener una obligación y estar obligado, ${ }^{93}$ afectando la propia noción de lo jurídico.

La proliferación de las coalitions of the willing no es más que una nueva expresión de esta vieja historia de tensiones entre legalidad y legitimidad, entre el derecho entendido como un proceso que regula su propia creación y como un orden concreto basado en la noción de lo políti-

93 Cfr. Hart, H. L. A., "Preface", The Concept of Law, 2a. ed., Oxford, Clarendon Press, 1997. 
co. ${ }^{94}$ Pero hemos aprendido mucho desde Weimar, y las Naciones Unidas no son, pese a todas sus deficiencias, la Sociedad de Naciones; sesenta años cuentan. Durante ese tiempo el derecho internacional ha superado crisis y se ha desarrollado enormemente, es decir, ha aprendido a autodeterminarse.${ }^{95}$ Sobre todo, en este tiempo no ha surgido, ni se vislumbra a futuro, otra fuente de legitimidad internacional que goce del mismo reconocimiento universal. En dicho sentido, creo que más que renegar de la existencia de las coalitions, tenemos que buscar las formas adecuadas para someterlas a un marco de legalidad. El derecho administrativo global ha empezado a elaborar el aparato conceptual necesario para analizar aquellos fenómenos que, como las coalitions, presentan serios desafíos a la doctrina de las fuentes del derecho internacional. Habrá que observar con detenimiento su evolución; por el momento, ya mostró que la construcción de un Estado internacional de derecho (international rule of law) no es únicamente una aspiración noble, sino también, y gracias a los avances de la dogmática jurídica, bastante realista.

94 Cfr. Koskenniemi, Martti, "Carl Schmitt, Hans Morgenthau...", cit., nota 24, pp. 17-19.

95 Cfr. Nolte, Georg, op. cit., nota 4. 